\title{
End of oversizing: smart designing of brake systems for BEVs
}

Dr. Jens Hoffmann, K. F. Wörsdörfer, Continental Teves AG \& Co. oHG

This manuscript is not available according to publishing restriction. Thank you for your understanding. 